\title{
Epidemiology of Clostridioides difficile infections, France, 2010 to 2017
}

Mélanie Colomb-Cotinat ${ }^{1,2}$, Laetitia Assouvie ${ }^{1,2}$, Julien Durand ${ }^{1}$, Côme Daniau ${ }^{1}$, Lucie Leon ${ }^{1}$, Sylvie Maugat ${ }^{1}$, Sophan Soing-

Altrach $^{1}$, Cécile Gateau ${ }^{3}$, Jeanne Couturier ${ }^{3}$, Isabelle Arnaud ${ }^{4}$, Pascal Astagneau ${ }^{4}$, Anne Berger-Carbonne ${ }^{1}$, Frédéric Barbut ${ }^{3}$

1. Santé publique France, Saint-Maurice, France

2. These authors contributed equally and share first authorship

3. National reference laboratory for anaerobic bacteria and C. difficile, St Antoine Hospital, Paris, France

4. Regional center for prevention of healthcare associated infections, Paris, France

Correspondence: Mélanie Colomb-Cotinat (melanie.colomb-cotinat@santepubliquefrance.fr)

Citation style for this article:

Colomb-Cotinat Mélanie, Assouvie Laetitia, Durand Julien, Daniau Côme, Leon Lucie, Maugat Sylvie, Soing-Altrach Sophan, Gateau Cécile, Couturier Jeanne,

Arnaud Isabelle, Astagneau Pascal, Berger-Carbonne Anne, Barbut Frédéric. Epidemiology of Clostridioides difficile infections, France, 2010 to 2017 . Euro Surveill. 2019;24(35): $\mathrm{pii}=1800638$. https://doi.org/10.2807/1560-7917.ES.2019.24.35.1800638

Article submitted on 26 Nov 2018 / accepted on o6 Jun 2019 / published on 29 Aug 2019

Background: Clostridioides difficile is a leading cause of healthcare-associated diarrhoea in middle and highincome countries. Up to 2018, there has been no systematic, annual surveillance for $C$. difficile infections (CDI) in France. Aims: To provide an updated overview of the epidemiology of CDI in France between 2010 and 2017 based on five different data sources. Methods: This is a descriptive study of retrospective surveillance and alerts data. Incidence of CDI cases was estimated through the CDI incidence survey (2016) and data from the French National Uniform Hospital Discharge Database (PMSI; 2010-16). Testing frequency for CDI was estimated through the CDI incidence survey and point prevalence studies on healthcare-associated infections (HAI; 2012 and 2017). The national early warning response system for HAI (HAl-EWRS, 2012-17) and National Reference Laboratory data (2012-17) were used to follow the number of severe CDI cases and/or outbreaks. Results: In 2016, CDI incidence in acute care was 3.6 cases per 10,000 patient days (PD). There was a statistically significant increase in CDI incidence between 2010 and 2016 (+14\% annually) and testing frequency was 47.4 per 10,000 PD. The number of CDI HAI-EWRS notifications decreased between 2015 and 2017 with only a few large outbreaks reported. Conclusion: The CDI incidence estimate increased from 2010, but remained below the European average of 7 per 10,000 PD in 2014; there were fewer severe cases or clusters reported in France. The consistency between PMSI and laboratory-based estimated CDI incidence could allow for more routine monitoring of CDI incidence.

\section{Introduction}

Clostridium difficile, officially renamed Clostridioides difficile in 2016 , is responsible for $15-25 \%$ of antibioticassociated diarrhoea cases $[1,2]$ and is considered the leading cause of healthcare-associated diarrhoea in developed countries. $C$. difficile infections (CDI) can be severe (toxic megacolon, septic shock) and represent one of the most expensive nosocomial infections [1,35]. In more than $95 \%$ of CDI cases, the patient receives antibiotics just before the onset of diarrhoea [2].

The epidemiology of CDI has changed over the past 20 years with the emergence of a hypervirulent clone NAP1/027/BI implicated in large outbreaks of severe CDI worldwide with high mortality rates [6-11] in the beginning of the 2000s. Between 2006 and 2007, this clone was responsible for outbreaks of severe CDI in the north of France before spreading gradually throughout the territory [9]; these outbreaks were controlledby the end of 2007. In 2009, following the epidemic period, a national prospective, multicentric survey to assess CDI incidence and to characterise CDI strains was launched in France (ICD-Raisin study). It showed an incidence of 2.28 CDI per 10,000 patient days (PD) in acute healthcare facilities (HCF) and 1.15 per 10,000 PD in long-term facilities [12]. The European, multicentric, prospective, point prevalence study (PPS) of CDI in hospitalised patients with diarrhoea (EUCLID) study, conducted in 2014 [13], found an incidence of 3.9 per 10,000 PD in 2011-12 in France and an estimated average incidence of 7 per 10,000 PD (range 0.7-28.7) in Europe.

Up to 2018, there has been no systematic, annual surveillance for CDI in France. Since 2007, routine surveillance of CDI is based on two data sources: (i) notifications by HCF through the national healthcareassociated infections early warning and response system (HAl-EWRS) [14], which are only mandatory for severe CDI presentations and/or outbreaks. Since 2012, the HAI-EWRS has been accessible via an online application (e-SIN), (ii) microbiological data from the national reference laboratory for $C$. difficile (NRL, Paris). NRL data do not reflect the overall epidemiology of $C$. difficile in France, however, as the strains usually 
Testing frequency and incidence of CDI in acute care by hospital type, France, CDI incidence survey 2016 ( $\mathrm{n}=203$ )

\begin{tabular}{|l|c|c|c|c|c|}
\hline Hospital type & $\begin{array}{c}\text { Number of } \\
\text { participating HCF }\end{array}$ & $\begin{array}{c}\text { Testing frequency per } \\
10,000 \text { PD }\end{array}$ & $\begin{array}{c}\text { Stools that tested positive } \\
\text { for CDI per 10,000 PD }\end{array}$ & $\begin{array}{c}\text { CDI cases per } \\
10,000 \text { PD }\end{array}$ & $\begin{array}{c}\text { HA CDI cases per } \\
10,000 \text { PD }\end{array}$ \\
\hline Tertiary & 12 & 52.8 & 6.1 & 4.7 & 2.7 \\
\hline Secondary & 100 & 51.8 & 4.6 & 3.6 & 2.0 \\
\hline Primary & 82 & 36.0 & 3.8 & 6.4 & 1.2 \\
\hline Specialised & 9 & 78.7 & 6.4 & 3.6 & 3.7 \\
\hline Total & 203 & 47.4 & 4.7 & 3.9 \\
\hline
\end{tabular}

CDI: Clostridioides difficile infection; HA: hospital acquired; HCF: healthcare facilities; PD: patient days.

${ }^{a}$ including oncology centres only.

come from severe cases or outbreaks and sending of the strains to $\mathrm{NRL}$ is not mandatory.

To complement and update available data on CDI in France, a laboratory-based CDI incidence survey was conducted in 2016 through the pre-existing national multidrug-resistant bacteria surveillance programme (French acronym BMR-Raisin) [15]. The survey was offered as an optional module for laboratories within acute HCF to complete. Two national PPS of HAI and antimicrobial use were also conducted in 2012 and 2017, which included data on CDI prevalence and the number of samples tested for $C$. difficile. In addition, information on inpatient hospital stays with CDI was collected from the French national hospital stays database (French acronym PMSI), for the 2010-16 period.

The aim of this study is to provide an updated overview of the epidemiology of CDI in France based on these five different data sources: (i) the 2016 CDI incidence survey, (ii) the 2012 and 2017 PPS, (iii) PMSI data, 2010-16, (iv) HAl-EWRS notifications, 2012-17 and, (v) NRL data, 2012-17. These five data sources have never been compared to each other, and only data from the PPS have been published elsewhere.

Of the five data sources, two have the purpose of alert: (i) notification to HAI-EWRS allows for a rapid real-time alert, communication between local team and regional or national support to help implementation of control measures, (ii) microbiological data from the NRL are used to follow the epidemic clone 027 and the potential emergence of more epidemic-prone or more virulent clones.

The purpose of the other three data sources are surveillance of CDI: (i) the CDI incidence survey, launched in 2016 , was useful in providing a point estimation of the CDI incidence in acute HCF, (ii) PPSs are done every 5 years in France and provide a point estimation on CDI prevalence and testing frequency in a representative sample of HCF in France, (iii) PMSI data were analysed for the first time at national level to estimate CDI incidence in acute HCF.

\section{Methods}

\section{The 2016 Clostridioides difficile infections incidence survey}

Source of information

Between April and June 2016, all laboratories within acute HCF in France were asked to complete an optional questionnaire including questions on: (i) algorithms used for CDI diagnosis, (ii) number of stool specimens tested for $C$. difficile, (iii) number of stool specimens that tested positive for CDI (there can be several for the same patient), and (iv) number of CDI cases and number of hospital-acquired CDI cases (HA CDI cases) for acute care wards within acute HCF. The questionnaire was in accordance with the European Centre for Disease Prevention and Control's (ECDC) technical document for minimal $C$. difficile surveillance [16]. Participation was voluntary.

A CDI case was defined as per European Society of Clinical Microbiology and Infectious Diseases (ESCMID) recommendations [9] i.e. diarrhoeal stools or toxic megacolon and a positive laboratory assay for $C$. difficile $\mathrm{TcdA}$ and/or TcdB in stools or a toxin-producing C. difficile organism detected in stools via culture or PCR. A HA CDI case was defined as a positive sample at least 48 hours following admission, with no manifest CDI infection in the 6 months before admission. Patients hospitalised for less than 24 hours and dialysis patients were excluded. The reference algorithms for the detection of a CDI were those recommended by the ESCMID [17].

\section{Analysis}

The testing frequency for CDI was estimated using the ratio between the number of stools tested for $C$. difficile to the number of PD in acute HCF over the study period (data source were the annual administrative 
Mean rates of Clostridioides difficile testing frequency and prevalence of patients diagnosed with C.difficile by hospital type, France, 2012 and 2017 PPS

\begin{tabular}{|c|c|c|c|c|c|c|c|c|c|}
\hline \multirow{3}{*}{ Hospital type } & \multirow{2}{*}{\multicolumn{2}{|c|}{$\begin{array}{c}\text { Testing frequency } \\
\text { per } 10,000 \text { PD } \\
\text { PPS } 2017\end{array}$}} & \multicolumn{7}{|c|}{ Prevalence of patients diagnosed with CDI } \\
\hline & & & \multicolumn{3}{|c|}{ PPS 2012} & \multicolumn{4}{|c|}{ PSS 2017} \\
\hline & Rate & $95 \% \mathrm{Cl}$ & $\begin{array}{l}\text { Number of } \\
\text { patients } \\
\text { included }\end{array}$ & $\begin{array}{c}\text { Number of } \\
\text { patients } \\
\text { diagnosed with } \\
\text { CDI }\end{array}$ & $\begin{array}{c}\text { Prevalence } \\
(\%)\end{array}$ & $\begin{array}{l}\text { Number of } \\
\text { patients } \\
\text { included }\end{array}$ & $\begin{array}{c}\text { Number of } \\
\text { patients } \\
\text { diagnosed with } \\
\text { CDI }\end{array}$ & $\begin{array}{c}\text { Prevalence } \\
(\%)\end{array}$ & $95 \% \mathrm{Cl}$ \\
\hline Tertiary & 68.11 & $\begin{array}{l}41.77^{-} \\
94.45\end{array}$ & 58,078 & 136 & 0.23 & 28,688 & 33 & 0.16 & $0.10-0.24$ \\
\hline Secondary & 39.82 & $37.03-42.61$ & 78,810 & 87 & 0.11 & 21,411 & 21 & 0.09 & $0.06-0.15$ \\
\hline Primary & 17.78 & $\begin{array}{c}15.24^{-} \\
20.32\end{array}$ & 94,568 & 79 & 0.08 & 17,338 & 16 & 0.10 & $0.06-0.16$ \\
\hline Specialised $2^{\mathrm{a}}$ & 58.74 & $N A^{b}$ & 2,267 & 1 & 0.04 & 978 & 3 & 0.25 & $0.08-0.84$ \\
\hline $\begin{array}{l}\text { Subtotal acute } \\
\text { HCF }\end{array}$ & $23 \cdot 48$ & $\begin{array}{c}20.73- \\
26.23\end{array}$ & 233,723 & 303 & 0.13 & 68,415 & 73 & 0.11 & $0,09-0,15$ \\
\hline Specialised $1^{c}$ & 3.79 & $2.8-4.78$ & 66,607 & 34 & 0.05 & 12,573 & 10 & 0.08 & $0.04-0.17$ \\
\hline Total & 15.86 & $4.13-17.59$ & 300,330 & 337 & 0.11 & 80,988 & 83 & 0.11 & $0.08-0.14$ \\
\hline
\end{tabular}

CDI: Clostridioides difficile infection; $\mathrm{Cl}$ : confidence interval; HCF: healthcare facilities; NA: not available; PD: patient days; PPS: point prevalence survey.

a Including oncology centres only.

b When there is only one primary sampling unit within a stratum, there is insufficient information to compute an estimate of that stratum's variance.

c Including psychiatric care, rehabilitation centres and long-term facilities.

survey of HCF statistics for 2016 [18]). The incidence of stools that tested positive for CDI and the incidence of HA CDI cases per 10,000 PD was estimated using the ratio between the number of stools that tested positive for CDI or HA CDI to the number of PD in acute HCF over the study period. We also calculated the proportion of HCF performing CDI diagnosis using one of the reference algorithms.

\section{The 2012 and 2017 point prevalence surveys}

\section{Source of information}

In the 2012 and 2017 PPS, data on all HAl (including CDI), treatments and risk factors were collected for all patients present on the hospital ward on the day of the survey. In 2012, the PPS was sent to all HCF in France and the participation rate was $75 \%(1,938 / 2,594)$. In 2017 , the PPS was conducted on a representative sample of 449 HCF (stratified by region and hospital type), with 403 (91\%) participating. The methodology of these PPS has been described elsewhere [19]. A patient infected with $C$. difficile was defined as a compatible clinical presentation (diarrhoeal stools or megacolon) with detection of toxins $A$ and $B$ in the stools or pseudomembranous colitis diagnosed after colposcopy or compatible histology at endoscopy or autopsy.

\section{Analysis}

The prevalence of patients infected with $C$. difficile (in 2012 and 2017) and the testing frequency (i.e. the number of stools samples tested for $C$. difficile in acute HCF per 10,000 PD, variable retrospectively collected in 2017 only, based on 2016 data) were analysed. As the 2017 PPS was only conducted on a representative sample of HCF, $95 \%$ confidence intervals $(\mathrm{CI})$ were calculated for the prevalence of patients with CDI. The prevalence of patients with CDI in 2012 and 2017 was compared using multilevel models (patient, HCF and region) with a Poisson regression (adjusting for age, sex, McCabe score, immunosuppression, urinary catheter, central venous catheter, peripheral venous catheter, respiratory assistance and hospital ward). Analyses were performed with Stata version 14.1 (StataCorp, College Station, Texas (TX), United States (US)).

\section{The French national uniform hospital discharge database data, 2010-2016}

\section{Source of information}

PMSI is a standardised national database describing all inpatient hospital stays [20] and is used for the production of standardised healthcare billing information and medical information concerning patients (comorbidities, age and sex). Pathologies are coded by principal diagnosis and optional associated diagnoses, by a clinician using the French version of the international classification of diseases 1oth Revision (ICD-10) [21]. The ICD-10 code A04.7 ' $C$. difficile enterocolitis' must be used for every patient with a CDI, left to the appreciation of the physician that coded the stays. 
Number and incidence of hospital stays with CDI, France, PMSI data 2010-2016

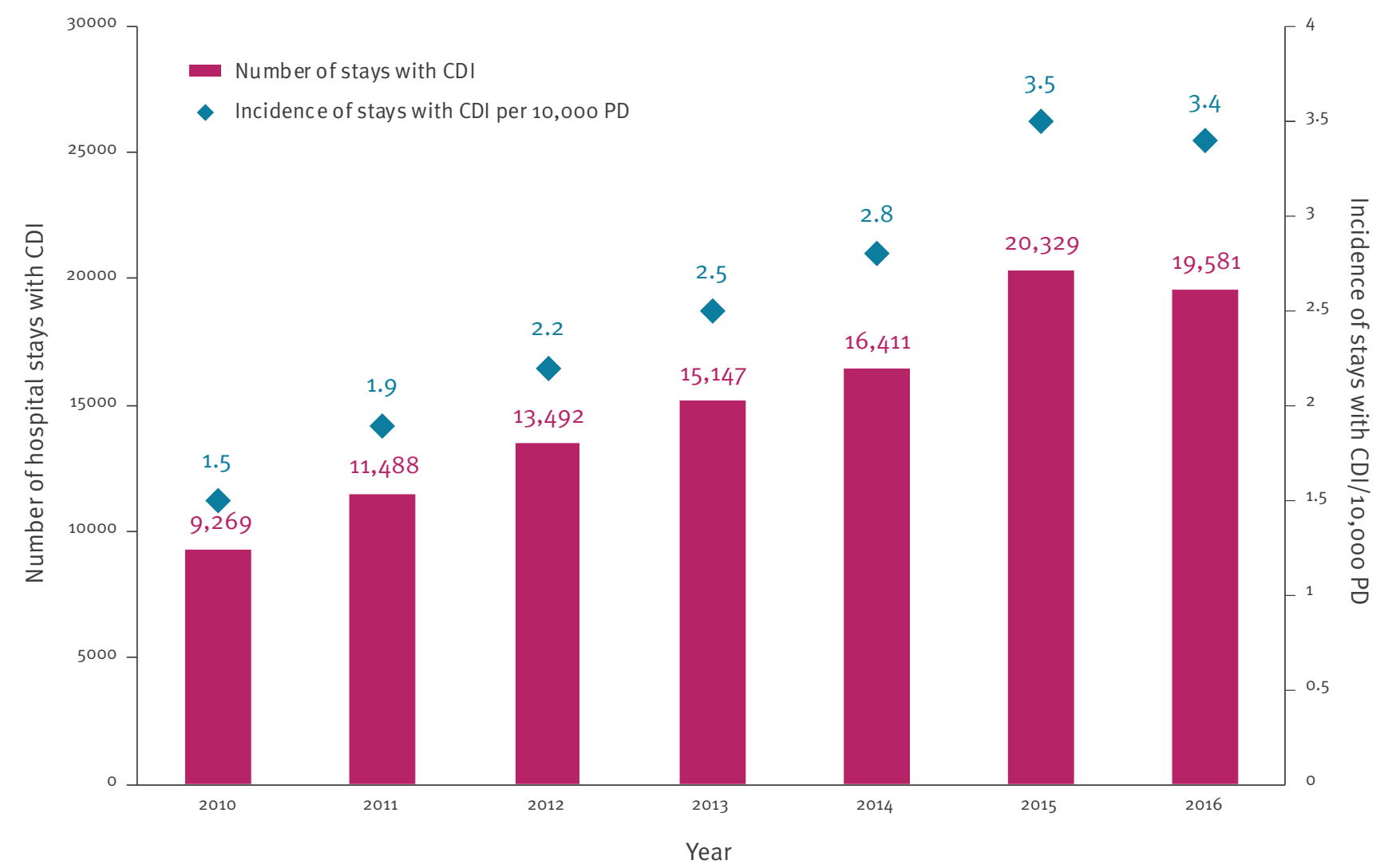

CDI: Clostridioides difficile infection; PD: patient days; PMSI: The French national uniform hospital discharge database.

Procedures are coded using a standardised classification (Classification Commune des Actes Médicaux [22]). Patients can have more than one stay in the same year.

\section{Analysis}

The PMSI was analysed for the period 1 January 201031 December 2016. Stays with an ICD-10 code A04.7 for principal or associated diagnosis were extracted from the PMSI database for acute wards in acute HCF and stratified by type of facility, type of stay, age, sex and region. The variable 'type of discharge' was used to identify in-hospital deaths (attributability was unknown). Stays, where a colectomy was performed, were identified with the following CCAM codes: HHFA002, HHFA004, HHFAO0 5, HHFA006, HHFAo08, HHFA009, HHFA010, HHFA014, HHFA017, HHFA018, HHFA021, HHFA022, HHFA023, HHFA024, HHFA026, HHFA028, HHFA029, HHFA030 or HHFA031. To calculate incidences, the ratio between the number of cases and stays to the number of PD (source: SAE 2016) were calculated. Regional data were estimated using the variable 'region of hospitalisation'. Incidences of stays with CDI between 2010 and 2016 were compared using Poisson regression with robust variance, adjusted for geographical region. Analyses were performed with Stata version 12 (StataCorp, College Station, TX, US).
Healthcare-associated infections early warning and response system notifications, 2012-2017

Source of information

The e-SIN database contains all healthcare-associated infections ( $\mathrm{HAl}$ ) notifications that have been reported since January 2012. Notifications report data on HCF (name and location) and data on date of detection of the first case, the number of cases and death, ward of hospitalisation, the microorganism responsible for infection and any control measures. A comment box for any additional information was available.

\section{Analysis}

HAI-EWRS notifications received between 1 January 2012-31 December 2017 were extracted from the e-SIN database. CDI notifications were identified based on the 'microorganism' item. The variables 'region', 'number of cases', 'number of deaths', 'infectious site' and 'ward' were extracted directly from the notifications. Ribotype of the strains was extracted from comments when necessary. An outbreak was defined as a notification reporting more than one case. Proportions of CDI notifications among HAl notifications between 2012 and 2017 were compared using a variance-weighted least-squares regression. Analyses were performed with Microsoft Excel 2013. 
A. 2010

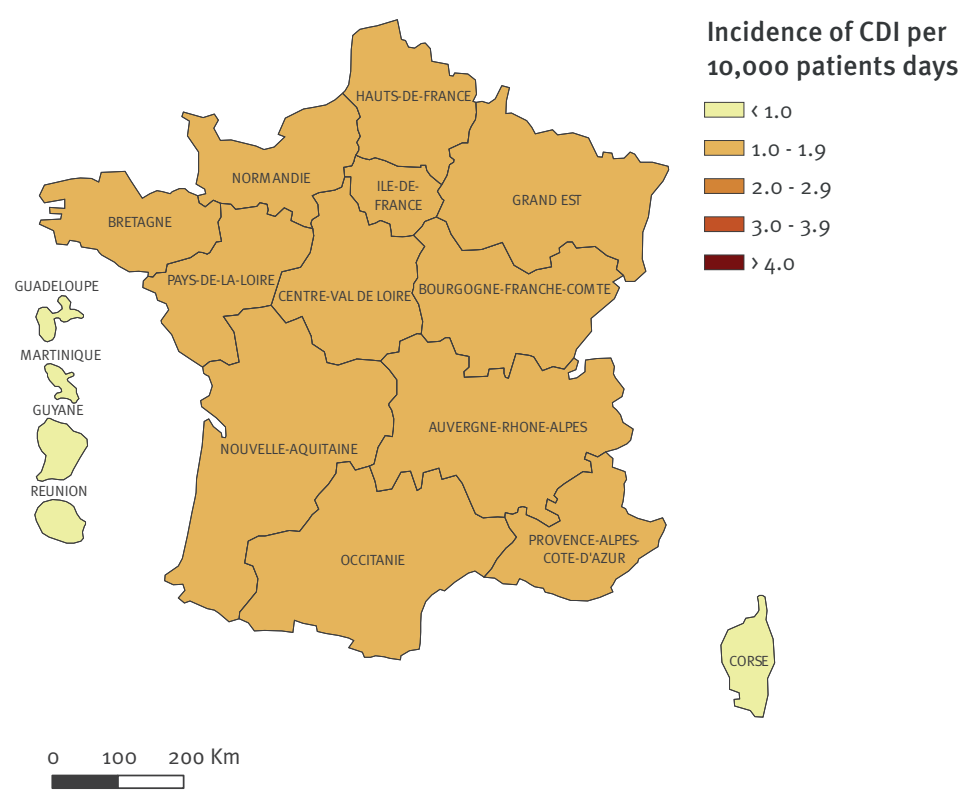

B. 2016

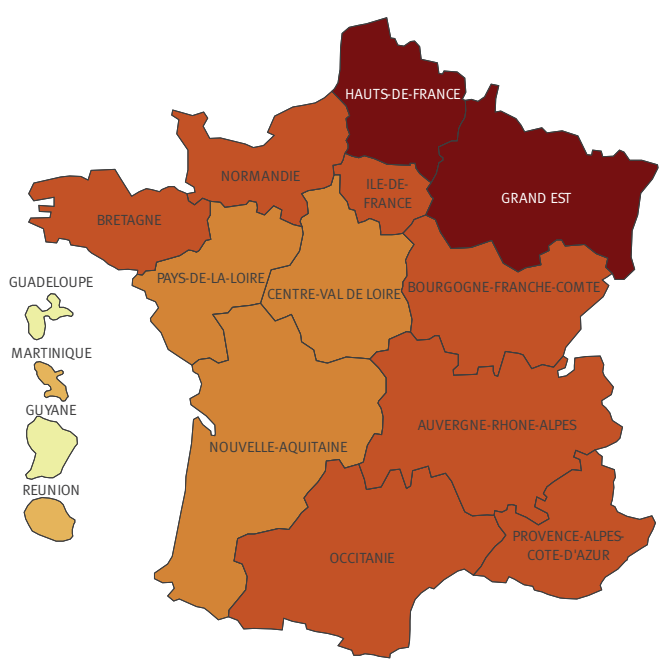

CDI: Clostridioides difficile infection; PSMI: The French national uniform hospital discharge database.

Source: Geofla région 2016, Santé Publique France.

National reference laboratory for Clostridioides difficile data, 2012-2017

\section{Source of information}

The NRL receives strains of $C$. difficile from voluntary French laboratories for characterisation. Most of these strains are linked to cases reported in the HAI-EWRS (clusters or severe forms of CDI). These strains are characterised by multiplex PCR which detects the main virulence factors (tcd $A$ and tcdB genes encoding toxins $A$ and $B$, respectively and $\operatorname{cdt} A$ and $c d t B$ genes encoding the binary toxin) and by capillary gel-based electrophoresis PCR ribotyping, as described elsewhere [23]. The strains' susceptibility patterns to antibiotics (metronidazole, vancomycin, erythromycin, tetracycline and moxifloxacin) are determined by the disk diffusion method. Antimicrobial susceptibility testing was performed according to the 2013 French CA-SFM (Comité de l'antibiogramme de la Société Française de Microbiologie) guidelines. Stain dilution (108 CFU/ml) is inoculated on Brucella Agar (Becton Dickinson) supplemented with vitamin $\mathrm{K}_{1}(1 \mathrm{mg} / \mathrm{ml})$ (Emprove, Merck), hemin $(5 \mathrm{mg} / \mathrm{L})$ (Applichem) and defibrinated horse blood (5\%). Plates are incubated $48 \mathrm{~h}$ at $35-37^{\circ} \mathrm{C}$, and diameters are interpreted according to the criteria for anaerobic bacteria given by the CA-SFM. C. difficile ATCC 700057 is used as quality control. The method was the same throughout the 5 years.

\section{Analysis}

Number and characteristic of strains analysed by the NRL between 2012 and 2017 were described.

\section{Ethical statement}

Anonymous surveillance data were collected from patient charts only for the public interest mission of the French public health agency or its partners, in accordance with the French data protection authority. Analyses were only conducted on aggregated data and not on an individual level.

\section{Results}

\section{The 2016 Clostridioides difficile infections incidence survey}

In 2016, of more than 2,000 acute HCF in France, 203 participated in the CDI incidence survey, corresponding to $10 \%(3,056,445 / 30,854,819)$ of total PD for the same year. All hospital types (tertiary, secondary and primary) and all 17 regions in France was represented. The testing frequency for CDI was 47.4 per 10,000 PD, while the incidence of stools that tested positive for CDI was 4.7 per 10,000 PD (positivity rate of 10\%). Diagnostic testing was performed using ESCMID-recommended algorithms in $65 \%$ of HCF. The incidence of CDI cases per 10,000 PD was 3.6, while the incidence of HA CDI cases was 1.9 (Table 1 ).

The 2012 and 2017 point prevalence surveys In 2012, of 300,330 patients included in the study, 337 had CDI (prevalence $=0.11 \%$ ). In 2017, of 80,988 patients included in the study, 83 had CDI (prevalence: $0.11 \% ; 95 \% \mathrm{Cl}: 0.08-0.14)$. After adjusting for the indicators of severity, the prevalence of patients 
FIGURE 3

Number of HAI-EWRS notifications and cases with CDI, France, 2012-2017

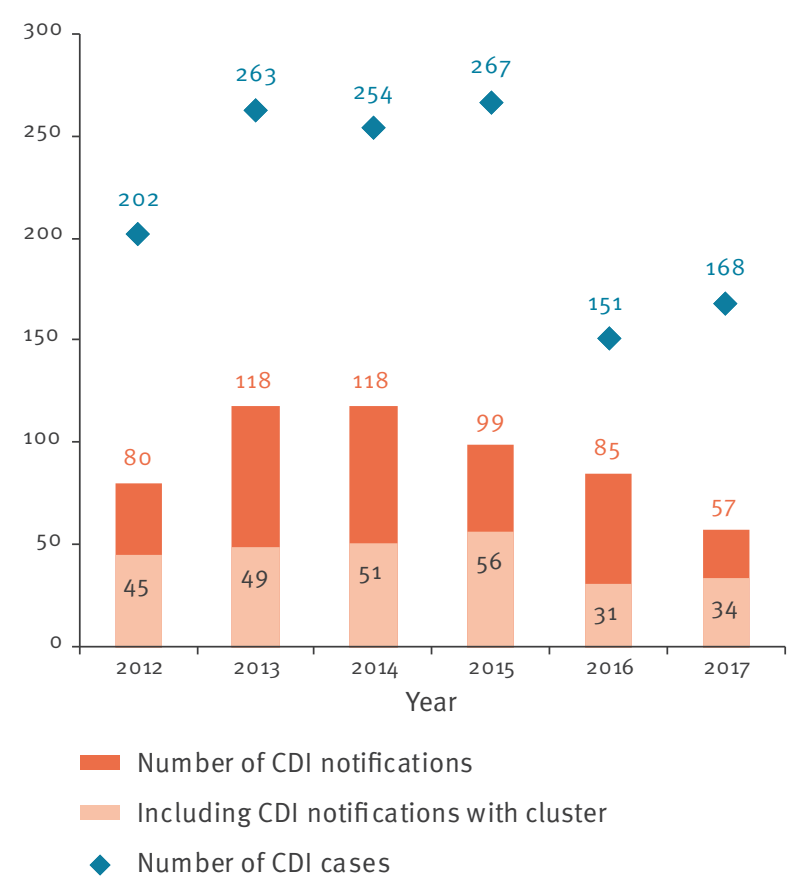

CDI: Clostridioides difficile infection; HAI-EWRS: HealthcareAssociated Infections Early Warning and Response System.

diagnosed with CDI remained stable between 2012 and 2017 ( $p>0.05)$.

In 2017, the mean rate of $C$. difficile testing frequency was 15.9 per 10,000 PD. Large differences were observed across the HCF categories (Table 2), with tertiary care hospitals having the highest rates. Most patients $(54 / 83 ; 65 \%)$ were hospitalised in secondary and tertiary hospitals (Table 2 ).

The French national uniform hospital

discharge database data, 2010-2016

Between 2010 and 2016, 86,953 patients were hospitalised in acute HCF in France with a coded diagnosis of CDI, corresponding to 105,717 stays. A steady increase was observed from 2010 to 2015 , followed by a slight decrease in 2016. The estimated incidence of stays with a CDI diagnosis significantly increased from 1.5 per 10,000 PD in 2010 to 3.4 per 10,000 PD in 2016 (+14\% per year ( $95 \% \mathrm{Cl}: 13-16)$, Figure 1$)$. This increase was observed in all French regions (Figure 2).

Of 86,953 patients hospitalised with $\mathrm{CDI}$ in acute HCF in France, 71,301 (82\%) had only one stay, 11,304 (13\%) had two and 4,348 (5\%) had more than two. Patients aged 80 years or older accounted for 31,717 $(36 \%)$ of patients, while those aged 14 years or less accounted for only 1,897 ( $2 \%)$. The sex-ratio was balanced in all age groups, except in patients aged over 80 years, where the proportion of women was higher (20,910/31,717; 66\% women).
CDI was coded as the principal diagnosis in $39 \%$ $(40,803 / 105,717)$ of stays. Of 64,914 stays where CDI was recorded as an associated diagnosis, the most frequent principal diagnoses were palliative care $(2,281$; $3.5 \%$ ). In total, 3,746 different principal diagnoses were listed.

The proportion of stays where death occurred was $12 \%$ $(12,614 / 105,717)$ and colectomies were performed in $1 \%$ of stays $(1,250 / 105,717)$. These proportions calculated for each year are stable during the study period. These proportions were also stable when the analysis was restricted to stays where $C$. difficile infection was coded as the principal diagnosis: $2,571 / 40,803(6 \%)$ patients died and 151/40,803 (0.4\%) colectomies were performed.

Healthcare-associated infections early warning and response system notifications, 2012-2017 A total of 557 notifications with $C$. difficile were received between 2012 and 2017, involving 1,305 patients and including 159 deaths (attributable or not). A decrease in the number of notifications with $C$. difficile was observed from 2015 onwards (Figure 3). The trend in the number of cases over time is more variable. Proportions of CDI notifications among all HAI notifications decreased by $1 \%$ annually $2012-17$ ( $p<10-5$, variance-weighted least-squares regression): $C D I$ notifications account for $5 \%(80 / 1,551)$ of HAI notifications in 2012 and $2 \%(57 / 2,890)$ in 2017.

Of 557 notifications of $\mathrm{CDI}, 166$ (30\%) occurred in rehabilitation/long-term care units and $56(10 \%)$ in geriatric units; a presumed or confirmed 027 strain of $C$. difficile was reported in $161(29 \%)$ of the notifications.

Among all CDI notifications, $245 / 557$ (44\%) reported at least two cases. This proportion varied between $36 \%$ and $60 \%$ depending on the year. The median number of cases per episode was three (range: 2-21). A few outbreaks involving more than 10 cases were reported: two in 2012, one in 2013, one in 2014, three in 2015 and three in 2017. No large outbreaks were reported in 2016.

National reference laboratory for Clostridioides difficile data, 2012-2017

Between 2012 and 2017, the frequency of PCR ribotype 027 and PCR ribotype 078/126 significantly decreased from $21.7 \%$ to $9.56 \%$ ( $p<0.0001$ ) and $12.9 \%$ to $7.49 \%$ $(p=0.02)$, respectively (Table 3$)$.

In 2017, of 387 C. difficile toxigenic strains, 25 (6.4\%) were the 'historical' PCR-ribotype 027 , susceptible to moxifloxacin and $99(25.7 \%)$ produced binary toxin. All strains were susceptible to metronidazole and vancomycin. The other PCR ribotypes remained relatively stable, except PCR ribotype $002(3.8 \%$ vs $8.01 \%)$ and PCR ribotype 106 ( $11 \%$ vs $4.65 \%$ ) which slowly emerged from 2012 to 2017. 
TABLE 3

Number of Clostridioides difficile strains analysed by the NRL, by ribotype, France, 2012-2017

\begin{tabular}{|c|c|c|c|c|c|c|c|c|c|c|c|c|}
\hline \multirow{3}{*}{ PCR-ribotype } & \multicolumn{12}{|c|}{ Number of strains (\%) } \\
\hline & \multicolumn{2}{|c|}{2012} & \multicolumn{2}{|c|}{2013} & \multicolumn{2}{|c|}{2014} & \multicolumn{2}{|c|}{2015} & \multicolumn{2}{|c|}{2016} & \multicolumn{2}{|c|}{2017} \\
\hline & $\mathrm{n}$ & $\%$ & $\mathrm{n}$ & $\%$ & $\mathrm{n}$ & $\%$ & $n$ & $\%$ & $\mathrm{n}$ & $\%$ & $n$ & $\%$ \\
\hline 27 & 91 & 21.7 & 126 & 23.8 & 67 & 13.5 & 75 & 14.0 & 60 & 15.9 & 37 & 9.6 \\
\hline 014/020/077 & 55 & 13.1 & 92 & 17.3 & 80 & 16.1 & 82 & 15.4 & 60 & 15.9 & 61 & 15.8 \\
\hline $078 / 126$ & 54 & 12.9 & 46 & 8.7 & 59 & 11.9 & 49 & 9.2 & 25 & 6.6 & 29 & $7 \cdot 5$ \\
\hline 002 & 16 & 3.8 & 22 & 4.1 & 33 & 6.6 & 27 & 5.1 & 23 & 6.1 & 31 & 8.0 \\
\hline 001 & 10 & 2.4 & 11 & 2.1 & 26 & 5.2 & 13 & 2.4 & 9 & 2.4 & 10 & 2.6 \\
\hline 005 & 9 & 2.1 & 10 & 1.9 & 11 & 2.2 & 24 & $4 \cdot 5$ & 15 & 3.9 & 0 & $<1$ \\
\hline 15 & 3 & $<1$ & 10 & 1.9 & 19 & 3.8 & 9 & 1.7 & 2 & 0.5 & 0 & $<1$ \\
\hline 17 & 5 & 1.2 & 8 & 1.5 & 4 & $<1$ & 5 & 0.9 & 0 & $<1$ & 1 & $<1$ \\
\hline 106 & 2 & $<1$ & 10 & 1.9 & 15 & 3 & 11 & 2.1 & 21 & 5.57 & 18 & 4.6 \\
\hline 53 & 0 & $<1$ & 2 & $<1$ & 4 & $<1$ & 0 & $<1$ & 0 & $\ll 1$ & 4 & 1.0 \\
\hline Other & 175 & 41.7 & 193 & 36.4 & 180 & 36.5 & 239 & 44.7 & 162 & 43.0 & 197 & 50.9 \\
\hline Total & 420 & 100 & 530 & 100 & 498 & 100 & 534 & 100 & 377 & 100 & 387 & 100 \\
\hline
\end{tabular}

NRL: national reference laboratory.

\section{Discussion}

This study has provided an updated overview of the epidemiological data available on CDI in acute HCF in France between 2010 and 2016, combining for the first time five different data sources. The CDI incidence survey, launched in 2016, was repeated in 2017 and 2018 but is time-consuming for laboratories and regional centres. The results of the 2017 and 2018 survey are not currently available (as at July 2019) and it will not be repeated after 2018. However, the CDI incidence in acute HCF in 2016 estimated using PMSI data were consistent with the incidence estimated from the CDI incidence survey conducted in the same year. A pilot study conducted in 2010 by the NRL [24] compared the sensitivity and specificity of a surveillance programme using PMSI data with laboratory-based surveillance data. It found that the PMSI data underestimated the incidence of CDI, compared with the laboratory results from the NRL (sensitivity 35.6\%). This suggests the PMSI coding has improved since 2010 and that PMSI data in 2016 could closely represent the true incidence of CDI cases - opening up the possibility for a national surveillance system utilising routine monitoring of the incidence of stays with CDI (PMSI data), complemented with microbiological-based surveillance carried out by the NRL. The ICD-10 code A04.7 is specific for CDI, making easy the use of PMSI for surveillance purpose. In addition, HAI-EWRS is still needed to help monitoring alerts and repeated PPS will provide a point estimation on CDI prevalence and testing frequency in a representative sample of HCF in France.

The 2016 CDI incidence survey estimated the CDI incidence in acute HCF as 3.6 cases per 10,000 PD, which is an increase from the 2.3 cases per 10,000 PD estimated in 2009 by the ICD-Raisin study. This increase may be due to greater awareness of $C$. difficile infection among clinicians and to the development of more sensitive diagnostic tests for $C$. difficile such as PCR. In France, only diarrhoeic stool samples and patients aged 3 years and older are recommended to be tested for $C$. difficile. Test-of-cure testing after a treatment for CDI and routine screening for colonisation on nondiarrheic stools are not recommended. Only a minority of laboratories in France systematically test for C. difficile in all diarrhoeic stool samples, the majority only do it when requested by a physician. Therefore, the testing frequency depends on a physician's awareness for C. difficile. CDI surveillance in England indicates that more than $75 \%$ of cases occur in patients aged 64 years and older [25]. Therefore, as the population is getting older and at a greater risk of CDI, an increase in incidence of CDI is possible [26].

Despite the increase of CDI in France (2010-16), the incidence of CDI in 2016 remains below the European 
average in 2014 (7/10,000 PD) [13], which could be explained by differences in CDI testing policies. The CDI incidence survey has estimated this $C$. difficile testing frequency at 47.4 stools tested per 10,000 PD in acute HCF in 2016, with differences regarding hospital type, while it was estimated at 62-69 stools tested per 10,000 PD on average in Europe [13]. This suggests that the proportion of stools tested for $C$. difficile is lower in France than in other European countries. The testing frequency estimated from PPS data were slightly different (23.48 stools tested/10,000 PD) and may be due to differences in participating acute HCF. In addition, data for the PPS also include long-term wards within acute HCF (lower testing frequency) whereas the CDIincidence survey excluded data from long-term wards. Further, participation in the CDI-incidence survey was voluntary so participating HCF may have been more aware of CDI as a result of outbreaks; this potential self-selection bias could explain the higher testing frequency, compared to the testing frequency estimated by the 2017 PPS, done on a representative sample of HCF.

In parallel, the number of CDI HAI-EWRS notifications reached a peak in 2013-14 (118 notifications/year) before decreasing from 2015 onwards. There was also a decrease in the number of 027 strains identified at the NRL. The proportion of HAl-EWRS notifications involving 027 strains varied widely from year to year, but this proportion also took into account possible 027 strains not confirmed by the NRL. In addition, some laboratories use the GeneXpert method, which does not have high specificity for the 027 strain leading to an overestimation. NRL microbiology data show the emergence of PCR ribotype 002 and PCR ribotype 106 from 2012 to 2017. PCR ribotype 002 has been shown endemic in some nursing homes in Hong-Kong [27] and PCR ribotype 106 has been responsible for outbreaks in vascular surgery [28].

The low number of CDI outbreak notifications in France is less worrying than in other countries such as Germany, where the epidemic clone accounts for $40 \%$ of $C$. difficile strains [29]. The prevention and control of CDI are based primarily on appropriate microbiological testing practices, antibiotics stewardship policy and prevention of cross-transmission by implementing contact precaution [30,31].

According to the PMSI data, the proportion of severe forms (i.e. leading to death or colectomy) remained stable over time, but we do not know whether death is attributable to CDI. Two studies have already shown excess mortality related to CDI compared with agematched and comorbidity-matched patients who did not have CDI $[4,32]$.

The majority of $C D I$ cases reported in our survey are $\mathrm{HA}$, which is not surprising as the survey targeted only hospitalised patients and C. difficile is one of the most common HA pathogen [33]. For example, the 2016
European epidemiological report of CDI [34] found that HA CDI made up $74.6 \%$ of cases. The increase observed in CDI-related hospital stays differed between regions in France. Regional incidence disparities should be studied in the future and maybe partly explained by disparities in healthcare organisation, especially in overseas regions (HCF activities, specialities etc.). Incidence of CDI-related hospital stays differed with the age of the patient. It would be interesting to estimate incidence in different age groups, and that will be the subject of further work.

A limitation of our study is the difference between CDI definitions between the data sources: (i) in the CDI incidence survey, definition is based on clinical elements and microbiological confirmation as recommended per the ESCMID, (ii) in the PSSs, definition is based on clinical or histological elements $+/$-detection of toxins in the stools, (iii) in the PMSI and HAI-EWRS, CDI case definition is decided by the physician coding the stays or making the notification, but should normally match the ESCMID definition as recommended in France.

\section{Conclusion}

This study which combines five different data sources on CDI epidemiology in France for the first time shows that despite an increase of CDI incidence between 2010 and 2016, the incidence of CDI cases in France in 2016 remains below the European average. There is a low number of CDI outbreak notifications and there is a decrease in the number of 027 strains analysed by the NRL. Surveillance and alert for CDI remains however essential and thanks to this study, we have opened up the possibility for a national surveillance system utilising routine monitoring of the incidence of stays with CDI (PMSI data), complemented with microbiologicalbased surveillance carried out by the NRL. In addition, further studies will be needed to estimate incidence in different age groups and to explore the difference in regional incidences.

\section{Acknowledgments}

The authors would like to thank the BMR-Raisin group for its involvement in the 2016 laboratory-based survey, and all participating healthcare professionals for their involvement in the CDI data production. Our thanks also to Aurélie Duarte and Stephane Le-Vu for their help, and to Jude Sweeney for the English revision of the manuscript.

\section{Conflict of interest}

None declared.

Authors' contributions

Mélanie Colomb-Cotinat and Laetita Assouvie and contributed equally to this article.

Mélanie Colomb-Cotinat conceived and designed the study, contributed to the interpretation of the results and wrote the manuscript; Laetitia Assouvie conducted the PMSI and 
HAI-EWRS data analysis and contributed to wrote the manuscript; Anne Berger-Carbonne conceived and designed the study, contributed to the interpretation of the results and revised the manuscript; Côme Daniau provided and analysed PPS data and contributed to the interpretation of the results; Julien Durand provided PSMI data and was involved in their analysis; Frédéric Barbut, Cécile Gateau and Jeanne Couturier provided and analysed LNR data, contributed to the interpretation of the results and the revision of the manuscript; Isabelle Arnaud and Pascal Astagneau provided and analysed the laboratory-based surveillance data and contributed to the interpretation of the results; Lucie Leon and Sylvie Maugat were involved in the PMSI data analysis; Sophan Soing-Altrach was involved in the HAI-EWRS data analysis.

\section{References}

1. CzepielJ, DróżdżM, PituchH, KuijperEJ, PeruckiW, MielimonkaA Clostridium difficile infection: review. Eur J Clin Microbiol Infect Dis. 2019;38(7):1211-21. DOI: 10.1007/s10096 019-03539-6 PMID: 30945014

2. HensgensMP, GoorhuisA, DekkersOM, KuijperEJ . Time interval of increased risk for Clostridium difficile infection after exposure to antibiotics. J Antimicrob Chemother. 2012;67(3):742-8. DOI: 10.1093/jac/dkr508 PMID: 22146873

3. GabrielL, Beriot-MathiotA. Hospitalization stay and costs attributable to Clostridium difficile infection: a critical review. J Hosp Infect. 2014;88(1):12-21. DOI: 10.1016/j.jhin.2014.04.011 PMID: 24996516

4. BarbutF, BouéeS, LongepierreL, GoldbergM, BensoussanC , Levy-BachelotL. Excess mortality between 2007 and 2014 among patients with Clostridium difficile infection: a French health insurance database analysis. J Hosp Infect. 2018;98(1):21-8. DOI: 10.1016/j.jhin.2017.07.006 PMID: 28712548

5. LefflerDA , LamontJT . Clostridium difficile Infection. N Engl J Med. 2015;373(3):287-8.PMID: 26176396

6. CoignardB, BarbutF, BlanckaertK, ThioletJM , Poujoll , CarbonneA Emergence of Clostridium difficile toxinotype III, PCR-ribotype 027-associated disease, France, 2006. Euro Surveill. 2006;11(9):E060914.1.PMID: 17075146

7. O'ConnorJR, JohnsonS, GerdingDN. Clostridium difficile infection caused by the epidemic BI/NAP1/027 strain. Gastroenterology. 2009;136(6):1913-24. DOI: 10.1053/j. gastro.2009.02.073 PMID: 19457419

8. PépinJ, ValiquetteL, AlaryME, VillemureP, PelletierA, ForgetK Clostridium difficile-associated diarrhea in a region of Quebec from 1991 to 2003: a changing pattern of disease severity. CMAJ. 2004;171(5):466-72. DOI: 10.1503/cmaj.1041104 PMID: 15337727

9. KuijperEJ , CoignardB , TüllP ESCMID Study Group for Clostridium difficile EU Member States European Centre for Disease Prevention and Control. Emergence of Clostridium difficile-associated disease in North America and Europe. Clin Microbiol Infect. 2006;12(6) Suppl 6;2-18. DOI: 10.1111/j.14690691.2006.01580.x PMID: 16965399

10. Deaths involving Clostridium difficile: England and Wales, 1999-2004. Health Stat Q. 2006; (30):56-60.PMID: 16759080

11. SmithA . Outbreak of Clostridium difficile infection in an English hospital linked to hypertoxin-producing strains in Canada and the US. Euro Surveill. 2005;10(6): E050630.2.PMID: 16783109

12. EckertC, CoignardB , HebertM , TarnaudC, TessierC, LemireA ICD-Raisin Working Group. Clinical and microbiological features of Clostridium difficile infections in France: the ICDRAISIN 2009 national survey. Med Mal Infect. 2013;43(2):6774. DOI: 10.1016/j.medmal.2013.01.004 PMID: 23498135

13. DaviesKA, LongshawCM, DavisGL, BouzaE, BarbutF, BarnaZ Underdiagnosis of Clostridium difficile across Europe: the European, multicentre, prospective, biannual, point-prevalence study of Clostridium difficile infection in hospitalised patients with diarrhoea (EUCLID). Lancet Infect Dis. 2014;14(12):120819. DOI: 10.1016/S1473-3099(14)70991-0 PMID: 25455988

14. Thiolet JM PI, Vaux S, Alleaume S, Coignard B. The French healthcare-associated infection early warning and response system: a tool for detecting and monitoring emerging infectious diseases in healthcare facilities. Bulletin épidémiologique hebdomadaire.2011;15-16-17:5

15. Santé publique France. Raisin. Réseau de surveillance des bactéries multirésistantes à partir des laboratoires de microbiologie BMR-Raisin - Guide méthodologique pour l'année 2016 [Monitoring Multidrug-Resistant Bacteria from Microbiology Laboratories: the BMR-Raisin netwok Methodological Guide for 2016]. Paris: Santé publique France; [accessed 30 Jul 2019]. French. Available from: https://www. santepubliquefrance.fr/content/download/5639o/file/bmrraisin-protocole-2016.pdf

16. European Centre for Disease Prevention and Control (ECDC). European Surveillance of Clostridium difficile infections. Surveillance protocol version 2.3. Stockholm: ECDC; 2017. Available from: https://ecdc.europa.eu/en/publicationsdata/european-surveillance-clostridium-difficile-infectionssurveillance-protocol-1

17. CrobachMJ, PlancheT, EckertC, BarbutF, TerveerEM DekkersOM European Society of Clinical Microbiology and Infectious Diseases: update of the diagnostic guidance document for Clostridium difficile infection. Clin Microbiol Infect. 2016;22(Suppl 4):S63-81. DOI: 10.1016/j. cmi.2016.03.010 PMID: 27460910

18. Ministére des Solidarités et de la Santé. Panoramas de la DRESS : les établissements de santé, édition 2018 [Panoramas of the DRESS: health facilities, 2018 edition]. Paris: Ministére des Solidarités et de la Santé; 2018. French. Available from: https://drees.solidarites-sante.gouv.fr/etudes-etstatistiques/publications/panoramas-de-la-drees/article/lesetablissements-de-sante-edition-2018.html

19. Thiolet JM, Vaux S, Lamy M, Gauthier A, Barret AS, Leon $L$, et al. Enquête nationale de prévalence des infections nososcomiales et des traitements anti-infectieux en établissements de santé, France, mai-juin 2012 [Point prevalence survey of healthcare-associated infections and antimicrobial use in French hospitals, May-June 2012 - Results]. 2013. French. Available from: https:// www.santepubliquefrance.fr/maladies-et-traumatismes/ infections-associees-aux-soins-et-resistance-auxantibiotiques/infections-associees-aux-soins/documents/ rapport-synthese/enquete-nationale-de-prevalence-desinfections-nosocomiales-et-des-traitements-anti-infectieux-enetablissements-de-sante-france-mai-juin-2012.-r

20. BoudemagheT, Belhadjl . Data Resource Profile: The French National Uniform Hospital Discharge Data Set Database (PMSI). Int J Epidemiol. 2017;46(2):392-392d. DOI: 10.1093/ije/dyw359 PMID: 28168290

21. World health Organization (WHO). International statistical classification of diseases and related health problems - 10th revision. Geneva: WHO; [accessed 30 Jul 2019]. Available from: https://www.who.int/classifications/icd/ICD1oVolume2 en_2010.pdf

22. Securite Sociale l'Assurance Maladie. Classification commune des actes médicaux.[Cited 30 Jul 2019]. French. Available from: https://www.ameli.fr/accueil-de-la-ccam/index.php

23. BarbutF, DayN, BouéeS, YoussoufA, GrandvoinnetL, LalandeV Toxigenic Clostridium difficile carriage in general practice: results of a laboratory-based cohort study. Clin Microbiol Infect. 2019;25(5):588-94. DOI: $10.1016 / j$. cmi.2018.12.024 PMID: 30616013

24. JonesG , TarightN , BoellePY, MartyJ, LalandeV , EckertC Accuracy of ICD-10 codes for surveillance of Clostridium difficile infections, France. Emerg Infect Dis. 2012;18(6):97981. DOI: 10.3201/eid1806.111188 PMID: 22607707

25. DjureticT, WallPG , BrazierJS . Clostridium difficile: an update on its epidemiology and role in hospital outbreaks in England and Wales. J Hosp Infect. 1999;41(3):213-8. DOI: 10.1016/ S0195-6701(99)90018-X PMID: 10204123

26. Institut national de la statistique et des etudes economiques (INSEE). Nathalie Blanpain et Guillemette Buisson. Population projections to 2070: twice as many people aged 75 and over as in 2013. INSEE premiere. 2016; $n^{\circ} 1619$. Abstract. Insee: Paris: 2016. Available from: https://www.insee.fr/en/ statistiques/2581288

27. LukS, HoAYM , ChanEHY, TsangIHL, NgTK, ToWK High Prevalence and Frequent Acquisition of Clostridium difficile Ribotype 002 Among Nursing Home Residents in Hong Kong. Infect Control Hosp Epidemiol. 2018;39(7):782-7. DOI: 10.1017/ ice.2018.92 PMID: 29733004

28. RatnayakeL, McEwenJ, HendersonN , NathwaniD , PhillipsG , BrownD Control of an outbreak of diarrhoea in a vascular surgery unit caused by a high-level clindamycin-resistant Clostridium difficile PCR ribotype 106. J Hosp Infect. 2011;79(3):242-7. DOI: 10.1016/j.jhin.2011.06.013 PMID: 21864938

29. van DorpSM , KinrossP, GastmeierP, BehnkeM, KolaA DelméeM European Clostridium difficile Infection Surveillance Network (ECDIS-Net) on behalf of all participants . Standardised surveillance of Clostridium difficile infection in European acute care hospitals: a pilot study, 2013. Euro Surveill. 2016;21(29):30293. DOI: 10.2807/1560-7917. ES.2016.21.29.30293 PMID: 27472820 
30. Haut conseil de la santé publique. Avis relatif à la maîtrise de la diffusion des infections à Clostridium difficile dans les établissements de santé français [Opinion on the control of the spread of Clostridium difficile infections in French health establishments]. Paris: Haut conseil de la santé publique; [Accessed 30 Jul 2019]. French. Available from: https://www. hcsp.fr/Explore.cgi/avisrapportsdomaine?clefr $=32$

31. Tschudin-Sutter S, Kuijper EJ, Durovic A, Vehreschild M,

Barbut F, Eckert C, et al. Guidance document for prevention of Clostridium difficile infection in acute healthcare settings. Clin microbial infect. 2018;24(10):1051-1054.

32. HensgensMP, GoorhuisA, DekkersOM, van BenthemBH , KuijperEJ. All-cause and disease-specific mortality in hospitalized patients with Clostridium difficile infection: a multicenter cohort study. Clin Infect Dis. 2013;56(8):1108-16. DOI: 10.1093/cid/cis1209 PMID: 23300235

33. CassiniA, PlachourasD, EckmannsT, Abu SinM, BlankHP, DucombleT Burden of Six Healthcare-Associated Infections on European Population Health: Estimating Incidence-Based Disability-Adjusted Life Years through a Population PrevalenceBased Modelling Study. PLoS Med. 2016;13(10): e1002150. DOI: 10.1371/journal.pmed.1002150 PMID: 27755545

34. European Centre for Disease Prevention and Control (ECDC). Healthcare associated infections: Clostridium difficile infections. ECDC. Annual epidemiological report for 2016. Stockholm, ECDC; 2018. Available from: https://ecdc.europa. eu/en/publications-data/healthcare-associated-infectionsclostridium-difficile-infections-annual

\section{License, supplementary material and copyright}

This is an open-access article distributed under the terms of the Creative Commons Attribution (CC BY 4.0) Licence. You may share and adapt the material, but must give appropriate credit to the source, provide a link to the licence and indicate if changes were made.

Any supplementary material referenced in the article can be found in the online version.

This article is copyright of the authors or their affiliated institutions, 2019. 